Journal of

Synchrotron

Radiation

ISSN 0909-0495

Received 17 October 2009

Accepted 15 December 2009

\section{One-dimensional small-angle X-ray scattering tomography of dip-coated polyamide 6 monofilaments}

\author{
P. Kraft, ${ }^{a *} \ddagger$ O. Bunk, ${ }^{\text {a }}$ F. A. Reifler, ${ }^{\text {b }}$ R. Hufenus, ${ }^{b}$ M. Heuberger ${ }^{b}$ and F. Pfeiffer ${ }^{c}$

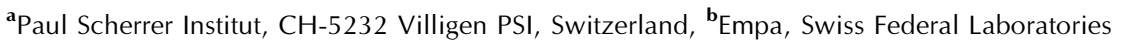 \\ for Materials Testing and Research, Laboratory for Advanced Fibers, Lerchenfeldstrasse 5, \\ CH-9014 St Gallen, Switzerland, and ${ }^{\mathbf{T}}$ Technische Universität München, James-Franck-Strasse, \\ 85748 Garching, Germany. E-mail: philipp.kraft@scs.ch
}

\begin{abstract}
A synchrotron study is presented in which the concept of one-dimensional tomographic reconstruction of small-angle X-ray scattering patterns is applied to investigate polyamide 6 monofilaments, dip-coated with alumina particles. The filaments are scanned with a focused synchrotron beam and the resulting scattering patterns are recorded with a PILATUS $2 \mathrm{M}$ detector. The reconstructed sequence of SAXS images reflects the local nanostructure variation along the filament radius. In particular, the influence of coating process parameters on the polyamide 6 is investigated.
\end{abstract}

(C) 2010 International Union of Crystallography Printed in Singapore - all rights reserved

Keywords: SAXS; one-dimensional tomography; polyamide 6; $\mathrm{Al}_{2} \mathrm{O}_{3}$; dip-coating; PILATUS; Abel transform; BASEX; two-point Abel deconvolution.

\section{Introduction}

Synchrotron beamlines featuring high brilliance, advanced optics and fast detectors are predestined for scanning microscopy experiments such as small-angle X-ray scattering (SAXS) tomography. SAXS tomography reveals information about the local nanostructure without destructive sample preparation. Based on the work presented by Schroer et al. (2006), scanning SAXS data from samples with axial symmetry is recorded at the cSAXS beamline of the Swiss Light Source (SLS) at the Paul Scherrer Institut (PSI). The goal is to test the feasibility of one-dimensional tomographic reconstruction by means of Abel inversion. Independently, this concept has been developed and evaluated by Stribeck et al. $(2008 a, b)$.

In the present work the aforementioned method is employed to study thick polymer monofilaments coated with ceramic particles. The two materials exhibit completely different nanostructures of which the characteristic SAXS can be disentangled by tomographic reconstruction such that radial structure variations in the polymer are unveiled. The data are recorded with the novel PILATUS 2M detector (Kraft et al., 2009) featuring single-photon counting and a maximum frame rate of $31.4 \mathrm{~Hz}$ for the full detector at $88.2 \%$ duty cycle, which improves data quality and data acquisition remarkably compared with standard charged coupled device (CCD) X-ray detectors. Although the beamline is still in the commissioning phase and not fully equipped at the time of the

$\ddagger$ Current address: Supercomputing Systems AG, Technoparkstrasse 1, CH8005 Zürich, Switzerland. measurements, a focused beam of about $20 \mu \mathrm{m}$ full width at half-maximum parallel to the filament and $5 \mu \mathrm{m}$ perpendicular to it is achieved which permits the sample to be scanned in $2 \mu \mathrm{m}$ steps with minor overlap. Instead of measuring the transmitted beam with an ionization chamber or a positiveintrinsic negative (PIN) diode inside the beamstop (Schroer et al., 2006), the attenuated direct beam is measured with the PILATUS $2 \mathrm{M}$ in a second scan, since modulation of the beam intensity at the SLS is relatively small owing to top-up injection.

\section{Experimental}

\subsection{Samples}

The studied samples are commercial polyamide 6 (PA6) monofilaments. ${ }^{\mathbf{1}}$ The diameter of filament 72 is $200 \mu \mathrm{m}$ whereas the diameters of filaments 73 and 74 are twice as much (see Table 1). The density of the PA 6 is provided by the manufacturer.

The monofilaments are treated in a dip-coating process with commercial alumina $\left(\mathrm{Al}_{2} \mathrm{O}_{3}\right)$ particles ${ }^{2}$ embedded in a polyester-urethane (PU) binder system (Reifler et al., 2008). The alumina particles are approximately spherical, have an average diameter of $2 \mu \mathrm{m}$ and a mass percentage with respect to the coating slurry of $39 \%$. The samples are amongst others

\footnotetext{
${ }^{1}$ Polyamide 6 monofilaments, type A172 by Monosuisse AG, Emmenbrücke, Switzerland (formerly Nexis Fibers AG, Emmenbrücke, Switzerland).

${ }^{2} \alpha-\mathrm{Al}_{2} \mathrm{O}_{3}$ Sumicorundum, high-purity alumina AA-2 [particle size: $D_{50}=$ $2.05 \mu \mathrm{m}$ (PIDS)] supplied by Sumitomo Deutschland GmbH, Düsseldorf, Germany.
} 
Table 1

List of measured PA 6 monofilaments.

\#: sample number; $D, \rho$ : diameter of monofilament and density of the PA6; $\mu_{\mathrm{PA} 6}^{\text {calc }}$ : calculated linear absorption coefficient of PA 6.

\begin{tabular}{llll}
\hline$\#$ & $D(\mu \mathrm{m})$ & $\rho\left(\mathrm{g} \mathrm{cm}^{-3}\right)$ & $\mu_{\mathrm{PA} 6}^{\text {calc }}\left(\mathrm{cm}^{-1}\right)$ \\
\hline 72 & 200 & 1.143 & 1.588 \\
73 & 400 & 1.155 & 1.604 \\
74 & 400 & 1.155 & 1.604 \\
\hline
\end{tabular}

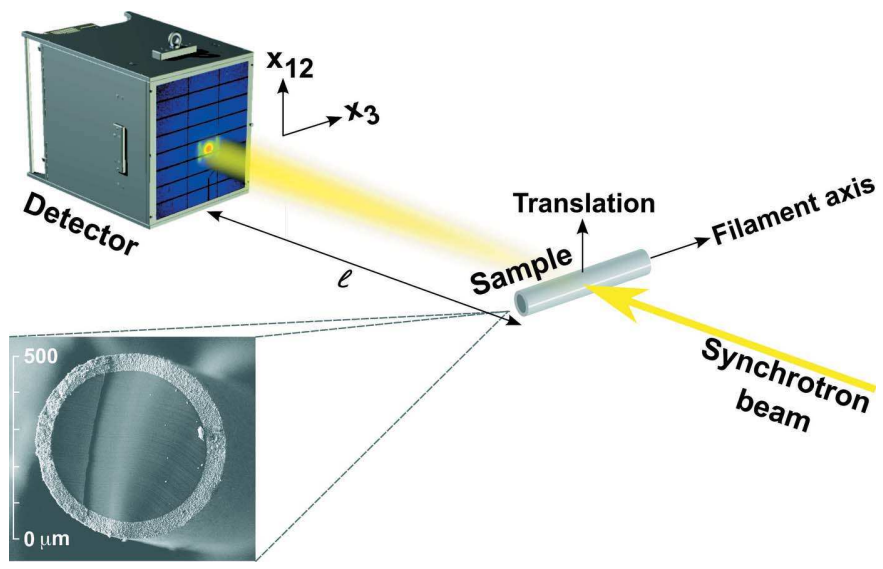

Figure 1

Scanning SAXS set-up. Inset: SEM image of a dip-coated PA6 monofilament cross section.

characterized by means of scanning electron microscopy (SEM) as shown in the inset of Fig. 1. The coating with ceramic microparticles is expected to improve the properties of the filament, such as higher resistance against heat, impact, cut, abrasion, hydrolysis and light while retaining the flexibility and elasticity (Gadow \& Von Niessen, 2002), as required in fabric, e.g. for ballistic protective or fireproof clothing.

The coating is accomplished with a custom-built dip-coating machine. After cleaning the filament with acetone it is transited through the slurry and subsequently dried and cured in two separate ovens. In order to find the optimum coating process parameters such that the covering is well adhering and mechanically resistive without losing the general properties of the pure filament, a set of filaments is dip-coated with varying withdrawal speeds and oven temperatures. The coating process parameters of filaments 72 and 73 are identical whereas filament 74 is processed with higher withdrawal speed and higher temperatures of the ovens (see Table 2). The higher withdrawal speed in the case of filament 74 results in a thicker coating. However, the coating thickness of filament 72 is smaller by $23 \%$ than the coating thickness of filament 73 although both are processed with identical coating parameters and only differ in diameter.

\subsection{Set-up}

The scanning microbeam SAXS experiments were carried out at the cSAXS beamline of the SLS at PSI, Switzerland. The basic measuring set-up is presented in Fig. 1. The incident beam (wavelength $\lambda=1 \AA$ ) is focused by means of the second
Table 2

Coating process parameters.

\#: sample number; $d$ : coating thickness; $T_{\mathrm{d}}$ : drying oven temperature; $t_{\mathrm{d}}$ : drying time; $T_{\mathrm{c}}$ : curing oven temperature; $t_{\mathrm{c}}$ : curing time; WS: withdrawal speed.

\begin{tabular}{lllllll}
\hline$\#$ & $\begin{array}{l}d \\
(\mu \mathrm{m})\end{array}$ & $\begin{array}{l}T_{\mathrm{d}} \\
(\mathrm{K})\end{array}$ & $\begin{array}{l}t_{\mathrm{d}} \\
(\min )\end{array}$ & $\begin{array}{l}T_{\mathrm{c}} \\
(\mathrm{K})\end{array}$ & $\begin{array}{l}t_{\mathrm{c}} \\
(\min )\end{array}$ & $\begin{array}{l}\mathrm{WS} \\
\left(\mathrm{m} \min ^{-1}\right)\end{array}$ \\
\hline 72 & 20 & 393 & 4 & 443 & 12 & 0.2 \\
73 & 26 & 393 & 4 & 443 & 12 & 0.2 \\
74 & 59 & 413 & 1.05 & 448 & 3.15 & 0.76 \\
\hline
\end{tabular}

monochromator crystal and a dynamically bendable mirror. The achieved footprint of the direct beam at sample level is investigated with a phosphor screen in front of a video camera with microscopic lens. The thereby determined beam widths are $20 \mu \mathrm{m}$ parallel to the filament axis and $5 \mu \mathrm{m}$ transversal to it. The distance between the sample mounted on a translation stage and the PILATUS $2 \mathrm{M}$ detector is $l=2.160 \mathrm{~m}$. The detector's threshold is set to $2 \AA$. A flight tube filled with helium and terminated with a mylar window is mounted between sample and detector to reduce parasitic scattering from gas molecules. The filament is moved perpendicular with respect to the filament axis in steps of $2 \mu \mathrm{m}$ and for each position a $1 \mathrm{~s}$ exposure is acquired. Each sample is measured twice. In the first data set the direct beam without beamstop is recorded using a silicon attenuator of thickness $2.4 \mathrm{~mm}$ before the sample to investigate beam stability (outside sample) and absorption (inside sample). The second data set contains the SAXS patterns using a beamstop after the sample and no beam attenuator. The parasitic scattering background of the set-up is determined with a few exposures in the SAXS configuration without sample.

\subsection{Preprocessing and reconstruction}

Owing to the axial symmetry and thermal gradients occurring radially inside a monofilament during its fabrication and the dip-coating process, radial structure variations in the PA 6 can be anticipated. This means that regions with the same radial distance from the filament center (sheath) are likely to exhibit an identical local nanostructure and a characteristic scattering pattern reflecting it. If the monofilament is probed with a microbeam, the illuminated volume includes mostly several sheaths. Hence, the resulting scattering contains contributions from different local structures. In order to glean the characteristic scattering for a volume element at a distinct position, a tomographic method is required. The axial symmetry of the sample and the assumptions made regarding the local structure allow for one-dimensional tomographic reconstruction by means of the inverse Abel transformation from a scattering data set of a single transversal microbeam scan (Stribeck et al., 2008a).

Basically the workflow as depicted by Stribeck et al. (2008b) is applied for preprocessing and tomographic reconstruction of the presented data. The beam center and the transmission for each position in the sample is determined from the center of gravity and integration of the direct beam images, respectively. The images are centered and cropped to a square of size 


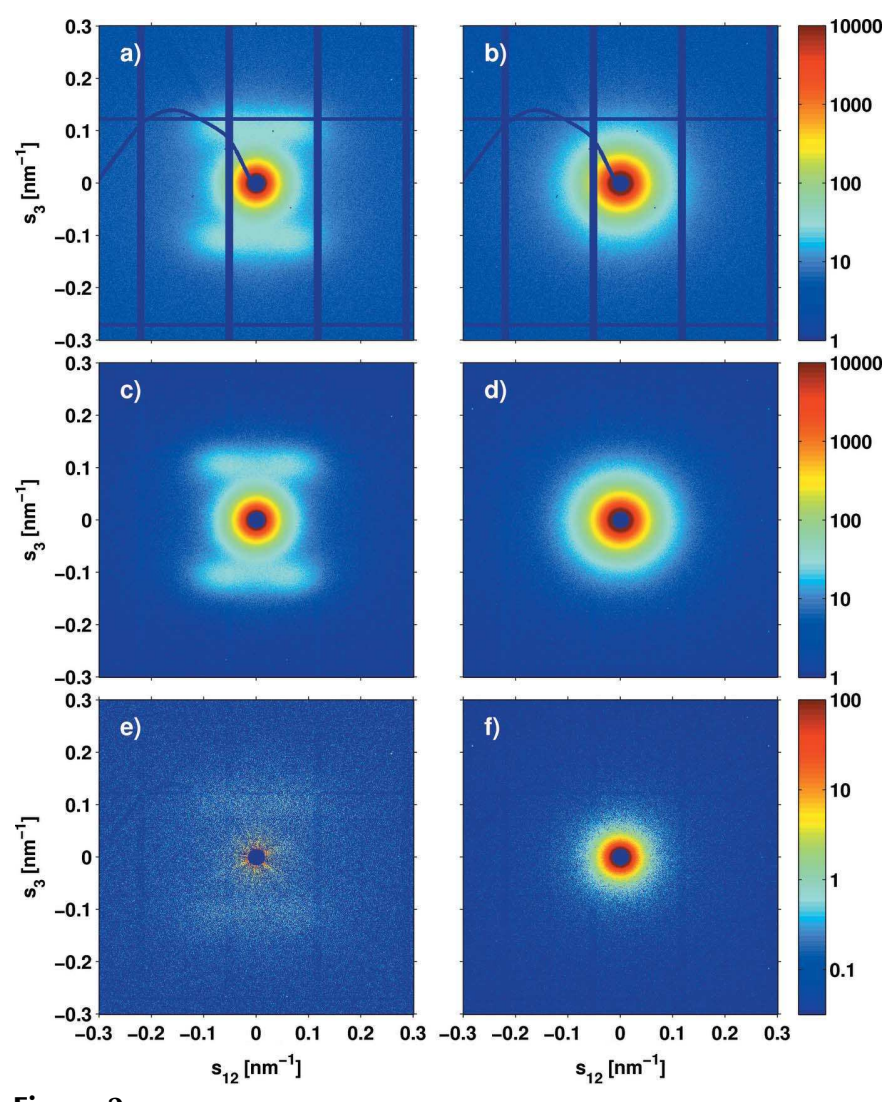

Figure 2

SAXS images of filament 74 (filament axis parallel to $s_{3}$ ). The left column corresponds to radius $r=40 \mu \mathrm{m}$, the right column to $r=212 \mu \mathrm{m}(r=0 \mu \mathrm{m}$ corresponds to the center of the filament). $(a),(b)$ Measured raw images; $(c),(d)$ preprocessed images; $(e),(f)$ reconstructed images.

$-0.3 \mathrm{~nm}^{-1} \leq s_{12}, s_{3} \leq 0.3 \mathrm{~nm}^{-1}$, where $s_{12}=x_{12} /(\lambda l), s_{3}=x_{3} /$ $(\lambda l), x_{12}$ denotes the distance on the detector from the beam center transverse to the filament axis and $x_{3}$ that parallel to the filament axis (see Fig. 1 and Figs. $2 a$ and $2 b$ ). From the shift of the direct beam center owing to refraction the exact transverse dimension and the center of the filament is determined. Owing to the filament symmetry, each image apart from the central one has a mirror image at the same radial distance from the center. Thus the two related mirror images are added to increase the counting statistics. The parasitic scattering background weighted by the corresponding transmission is subtracted from each SAXS image. Dead pixels and the gaps between detector modules are filled exploiting the centrosymmetry of the SAXS patterns (see Figs. $2 c$ and $2 d$ ). The area of the beamstop is left unaltered since tomographic reconstruction and the subsequent data analysis are unaffected by it.

The one-dimensional tomographic image reconstruction is accomplished by a matrix-vector multiplication. Both recommended algorithms are employed: the two-point Abel deconvolution (Dasch, 1992) and the BASEX Abel transform method (Dribinski et al., 2002).

\section{Results and discussion}

All recorded SAXS patterns exhibit isotropic scattering around the beamstop. In addition, a four-point pattern appears in the images taken within the PA6. The tomographic reconstruction reveals that the isotropic scattering is generated in the coating whereas the four-point pattern stems from the structure of the PA 6 (see Figs. $2 e$ and $2 f$ ). Neither belownor ultra-reconstruction artefacts were noticed in the reconstructed SAXS data (Stribeck et al., 2008a).

\subsection{Long period of the PA6}

Since the scattering of PA 6 is weak, the short exposure time leads to a low signal-to-noise ratio in the reconstructed SAXS patterns. Thus, automated analysis by means of the interface distribution function or the multidimensional chord distribution function (Stribeck, 2001, 2007) is not viable. However, the determination of the long period from the four-point pattern maxima, $L_{12}=\left|1 / s_{12 L}\right|$ and $L_{3}=\left|1 / s_{3 L}\right|$, is found to be feasible. For this purpose the patterns are projected on the $s_{3}$ axis and the $s_{12}$ axis, respectively (Stribeck, 2000). The central scattering has to be masked in the case of the $s_{12}$ projection in order to render the four-point scattering feature clearly visible. For each resulting scattering curve the monotonously decaying background is smoothed outside of the feature and interpolated under it. The feature itself is smoothed and the background subtracted yielding a single peak whereby determination of the long period is possible (Stribeck, 2007). None of the two applied reconstruction algorithms proves in this case to be notably less advantageous regarding the resulting data quality.

The observed four-point SAXS pattern from PA6 can be explained by two different structural models (Fronk \& Wilke, 1985; Stribeck et al., 2008a). On the one hand a macro-lattice of block stacks with a longitudinal distance $L_{3}$ between the hard-domain blocks is considered to yield such a pattern. The block stacks can be regarded as microfibrils consisting of linearly alternating hard and soft domains, with a transverse distance between the microfibrils of $L_{12}$ (see Fig. $3 a$ ). Description of the PA 6 fiber structure by this particular model is proposed by Bukošek \& Prevoršek (2000). On the other hand a four-point SAXS pattern can be due to a system of tilted lamellar stacks with a long period of $L=\left(s_{12 L}^{2}+s_{3 L}^{2}\right)^{-2}$ between lamellae and a tilt angle with respect to the filament

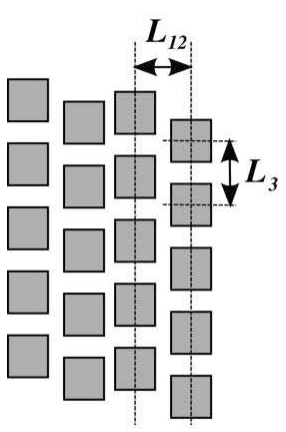

(a)

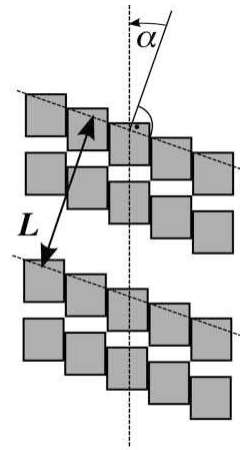

(b)
Figure 3

Structure models for PA 6 filaments: $(a)$ microfibrils, $(b)$ tilted lamellar stacks. After Fronk \& Wilke (1985). 
axis of $\alpha= \pm \arctan \left(L_{3} / L_{12}\right)$ (see Fig. $3 b$ ). This model is also applied by Murthy et al. (1997) to describe the structure of PA 6 fibers. None of the aforementioned structural models can be excluded owing to the low signal-to-noise ratio in the present SAXS data. Even a core-sheath structure (Iwata et al., 2006) with two kinds of conformations is conceivable. Hence the structural parameters $L(r)_{12}, L(r)_{3}$ for the microfibrils model and the accordingly computed parameters $L(r), \alpha(r)$ for the tilted lamellar stacks model are presented in Fig. 4 . The diameter of the PA 6 strand of filament 72 is only half that of the other two filaments. Thus the scattering, in particular in the reconstructed data, is weaker which is reflected in the stronger modulation of the determined long periods. Considering the microfibrils model (see Fig. $3 a$ ), the obtained average long periods ( $L_{12} \simeq 17 \mathrm{~nm}, L_{3} \simeq 9 \mathrm{~nm}$ ) are assumed to be of the order of the crystallite size. This is affirmed by measured PA6 crystal heights determined from wide-angle X-ray diffraction (Vasanthan, 2007) which are comparable with the present long periods.

The parameter $L(r)_{12}$ obtained from measured non-reconstructed data is notably increasing for larger radii, which is observed for all samples (see Fig. 4). This behavior is not reflected in the reconstructed data and thus is not attributable to the PA 6 structure. But it can be explained by the increase of isotropic scattering background towards larger radii which is subtracted from the four-point pattern (see $\$ 2.3$ ), meaning that systematic errors owing to smoothing and interpolation can bias the peak maximum in non-reconstructed data notably. The isotropic background becomes so dominant that the automatic algorithm even fails to determine a proper peak maximum 20 to $30 \mu \mathrm{m}$ before the position at which the beam leaves the PA 6 domain. However, the parameter $L(r)_{3}$ from non-reconstructed data is not remarkably differing from the same parameter determined from reconstructed data.

The long periods $L(r)_{12}$ obtained from reconstructed data are very similar for filaments 72 and 73, namely a slight increase in $L(r)_{12}$ beginning at $r=20$ to $30 \mu \mathrm{m}$ before the transition to the coating (see Fig. 4). This can be explained by the following considerations. Heating-up of the PA 6 can lead to additional crystallization and thus to larger crystallites resulting in a remarkable change in structure parameters (Bukošek \& Prevoršek, 2000). Heat absorption of the PA6 during the coating process is significantly higher for filaments 72 and 73 since their coating is thinner and the drying and curing times are 3.8 times longer, whereas thermally induced crystallization takes place at temperatures above $423 \mathrm{~K}$ (Vasanthan, 2004). Thus, it is likely that sufficient temperatures are reached in the PA6 which permit crystal growth during the curing process. Since the resulting temperature gradient points from the filament center to the surface, crystallites at higher filament radii grow more compared with crystallites closer to the filament center. Alternatively, the
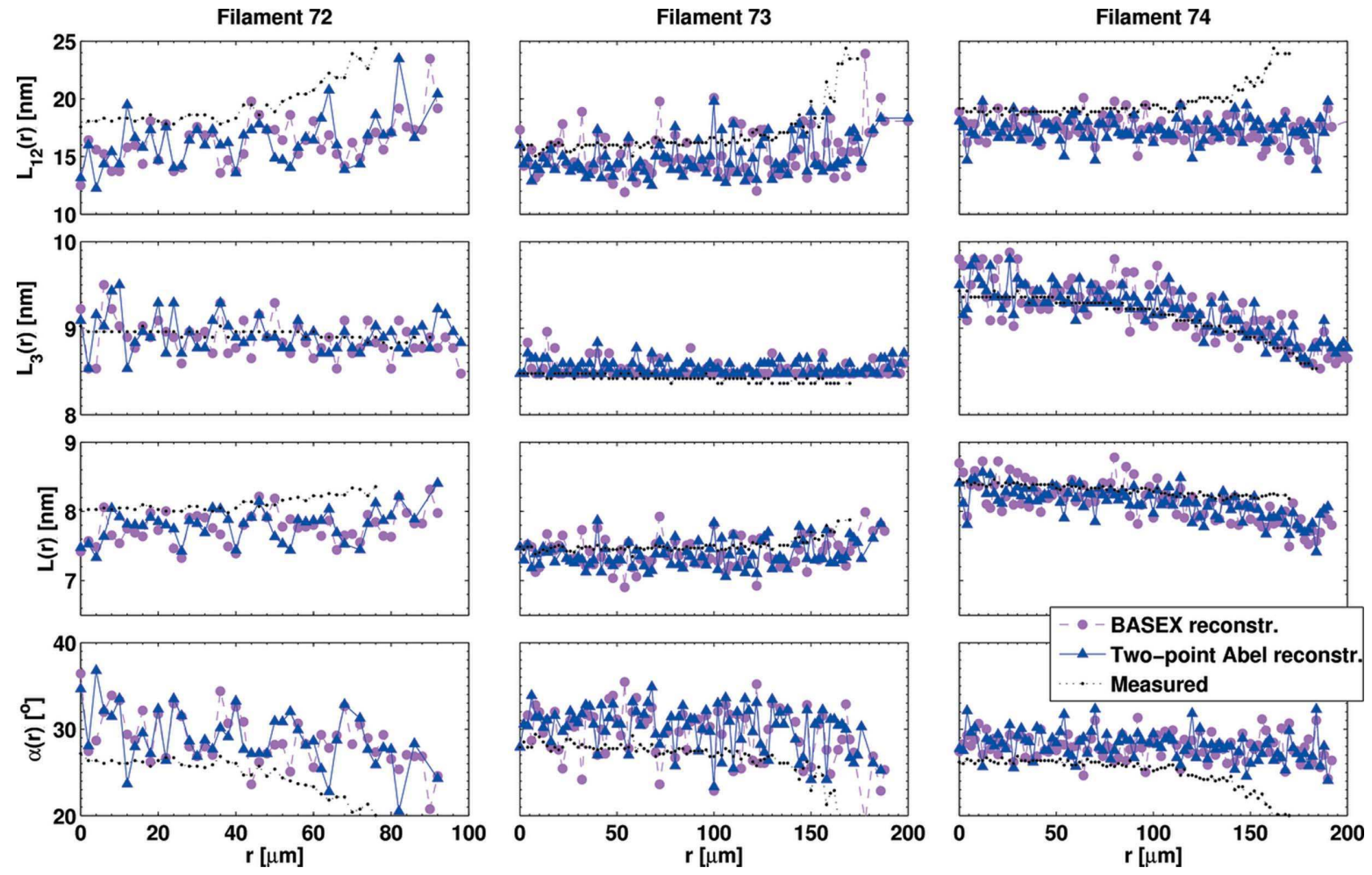

Figure 4

Structure parameters from the four-point pattern according to the microfibrils $\left[L(r)_{12}, L(r)_{3}\right]$ and the tilted lamellae structural model $[L(r), \alpha(r)]$, respectively, are plotted with respect to the radius inside the PA 6 monofilaments $(r=0 \mu \mathrm{m}$ corresponds to the center of the filament). The parameters are extracted from tomographically reconstructed SAXS patterns using the BASEX method and the two-point Abel inversion algorithm for reconstruction. For comparison, the same parameters extracted from the measured SAXS patterns are shown. The PA 6 monofilaments 73 and 74 have twice the diameter of filament 72 (see Table 1), but filament 74 has been dip-coated with higher withdrawal speed and higher oven temperatures (see Table 2). Missing data points are caused by failure of the automated analysis. 
observed increase in $L(r)_{12}$ close to the PA6 surface is explicable with a crystal-to-crystal transition which has been demonstrated to occur at temperatures of $433 \mathrm{~K}$ (Vasanthan, 2004).

By contrast, $L(r)_{12}$ obtained for filament 74 stays constant. The thick coating of filament 74 isolates its PA 6 well during the drying and curing process. Furthermore, the withdrawal speed is higher compared with filaments 72 and 73 which results in shorter drying and curing times. This results in minor heat absorption of the PA6. Hence, the nanostructure of filament 74 is expected to remain almost unaltered and thus represents best the state after the filament production, e.g. before the coating process.

During the filament production the heat dissipation at the surface of the PA 6 filament results in a temperature gradient from the surface towards the filament center. This leads to a decreasing crystallite size in the opposite direction. Thus, the decrease in $L(r)_{3}$ towards the PA 6 surface of filament 74 can be explained by the decrease in crystallite size owing to production. Assuming that $L(r)_{3}$ of filaments 72 and 73 originally followed the same trend as $L(r)_{3}$ of filament 74 after filament production, it could be ascribed to the coating process that the expected decrease in $L(r)_{3}$ towards the PA 6 surface is cancelled out in filaments 72 and 73 .

The aforementioned considerations apply analogously for the parameters $L(r)$ and $\alpha(r)$ of the tilted lamellae structure model.

\subsection{Linear absorption coefficient}

The linear absorption coefficient $\mu_{\text {PA6 }}$ can be calculated from the total formula of PA $6\left(\mathrm{C}_{6} \mathrm{H}_{11} \mathrm{NO}\right)$, the density provided by the manufacturer and the wavelength of the radiation (see Table 1). The transmission measured when the direct beam travels through the center of the filament is given by $T_{\text {center }}=\exp \left[-\left(\mu_{\mathrm{PA} 6} D+\mu_{\mathrm{c}} 2 d\right)\right]$ with $D$ being the diameter of the PA 6 strand, $d$ the thickness of the coating and $\mu_{\mathrm{c}}$ the linear absorption coefficient of the coating. Therefore, $\mu_{\mathrm{c}}$ is readily obtained (see Table 3 ) utilizing the values given in Table 1 and the measured absorption $T_{\text {center }}$. This method for determination of $\mu_{\mathrm{c}}$ is found to be the most robust.

By means of He pycnometry the volume of a solid can precisely be detected by measuring the amount of displaced helium gas by the solid. Successive weighing of the same specimen permits computation of its density. The densities of the alumina powder and of the coating (with and without alumina powder) have been determined using this technique (Sánchez, 2007). The obtained densities permit the calculation of the density of the alumina proportion in the coating. Thus the linear absorption coefficient for X-ray radiation can be computed for this proportion $\left(\mu_{\mathrm{c}}^{\text {calc }}=17.04 \mathrm{~cm}^{-1}\right)$. Although this value considers only the alumina proportion, it approximates the real value closely owing to the low absorption power of the PU matrix compared with the alumina particles. The exact composition of the commercial PU-based binder system is not known and hence the calculation of its linear absorption coefficient is impossible.
Table 3

The average dimensionality parameter obtained from the isotropic scattering generated in the coating, and the linear absorption coefficient of the coating $\mu_{\mathrm{c}}$ determined from the absorption measured at the filament center.

\begin{tabular}{lll}
\hline$\#$ & Dimension $a$ & $\mu_{\mathrm{c}}\left(\mathrm{cm}^{-1}\right)$ \\
\hline 72 & 3.96 & 18.0 \\
73 & 3.96 & 16.9 \\
74 & 3.98 & 15.6 \\
\hline
\end{tabular}

\subsection{Dimensionality of aluminium particles}

Determination of the average size of the alumina particles by means of the Guinier law requires data in the ultra SAXS regime and is thus impossible with the data at hand. However, the dimensions of the particles can be determined from the asymptotic form of the azimuthally integrated intensity $(I)$ curves by the relation $\ln (I) \propto-a \ln (s)$, where $s=\left(s_{12}^{2}+s_{3}^{2}\right)^{1 / 2}$ (Roe, 2000). The fitted slope parameter ${ }^{3} a$, which is for all samples close to 4 (see Table 3), indicates a spherical shape of the alumina particles.

\section{Conclusions}

Scanning SAXS data recorded across dip-coated PA6 exhibiting axial symmetry are reconstructed tomographically. The method is successfully applied and the subsequent data analysis reveals parameters indicating the local nanostructure in the PA6. Comparison with results in the literature discloses coincidence with two models which have been applied earlier to describe the structure of PA 6. Notable local trends in those parameters indicate an influence of the heat treatment during the dip-coating process on the nanostructure of the filaments. To reliably attribute this observation to the influence of postprocessing, ideally both a processed and an unprocessed sample should be measured for comparison. Furthermore, the dimensionality of the alumina particles in the coating is determined and the linear absorption coefficient for the coating derived.

Therefore, we conclude that the presented method permits investigation of the influence of the dip-coating process on the morphology and structure of a monofilament. The gained results could then be used to optimize the dip-coating process regarding the filament structure. Moreover, this method is suitable for studying and improving the morphology and local structure of filaments in general with respect to composition (e.g. bicomponent fibers), production conditions and processing.

Apparently SAXS images for one-dimensional tomography require better statistics with respect to the presented data in order to ease automated analysis, to extract more information and to achieve better accuracy of the obtained structural parameters. An estimated exposure time of 5 to $10 \mathrm{~s}$ per image instead of $1 \mathrm{~s}$ should provide sufficient intensity from the

\footnotetext{
${ }^{3}$ The results for SAXS images taken in the coating region are independent of the tomographic reconstruction, since the scattering is in both cases generated by the coating only.
} 
weakly scattering PA6. Consequently, the overall measurement period of a sample is increased. On the other hand, an increase in the scanning step size, and acquisition of images from the filament center to one sample edge only, could be considered to shorten the experiment without remarkable loss of information.

Measuring the attenuated direct beam with a positionsensitive detector reveals additional information such as the beam deflection. In particular, the reconstruction of the sample's local phase shift from the beam deflection, as it is obtained from filtered back projection tomography of differential phase contrast images (Pfeiffer et al., 2007), seems a possible extension of the presented technique. The method of deriving the unknown linear absorption coefficient of the coating from the transmission measured in the filament center works well, since the obtained values agree soundly with the calculated approximation. Unfortunately this method is only applicable if one of the two linear absorption coefficients is known.

We would like to thank F. A. L. Sanchéz for dip-coating the monofilaments. We gratefully acknowledge Dr N. Stribeck for making all of his processing functions available on the web. Provision of the BASEX algorithm in the form of a MATLAB function by Dr H. Reisler and Dr B. Karpichev is acknowledged.

\section{References}

Bukošek, V. \& Prevoršek, D. C. (2000). J. Polym. Matter, 47, 569592.
Dasch, C. J. (1992). Appl. Opt. 31, 1146-1152.

Dribinski, V., Ossadtchi, A., Mandelshtam, V. A. \& Reisler, H. (2002). Rev. Sci. Instrum. 73, 2634-2642.

Fronk, W. \& Wilke, W. (1985). Colloid Polym. Sci. 263, 97-108.

Gadow, R. \& Von Niessen, K. (2002). Ceramic Engineering and Science Proceedings 23, edited by H.-T. Lin and S. Singh, pp. 277285, Westerville, Ohio, USA.

Iwata, T., Aoyagi, Y., Tanaka, T., Fujita, M., Takeuchi, A., Suzuki, Y. \& Uesugi, K. (2006). Macromolecules, 39, 5789-5795.

Kraft, P., Bergamaschi, A., Broennimann, Ch., Dinapoli, R., Eikenberry, E. F., Henrich, B., Johnson, I., Mozzanica, A., Schlepütz, C. M., Willmott, P. R. \& Schmitt, B. (2009). J. Synchrotron Rad. 16, 368-375.

Murthy, N. S., Zero, K. \& Grubb, D. T. (1997). Polymer, 38, $1021-$ 1028.

Pfeiffer, F., Bunk, O., Kottler, C. \& David, C. (2007). Nucl. Instrum. Methods Phys. Res. A, 580, 925-928.

Reifler, F., Clemens, F., Sánchez, F. A. L., Varga, K. \& Hufenus, R. (2008). Fiber Society Spring Conference, pp. 133-134, Mulhouse, France.

Roe, R. J. (2000). Methods of X-ray and Neutron Scattering in Polymer Science. Oxford University Press.

Sánchez, F. A. L. (2007). Unpublished results.

Schroer, C. G., Kuhlmann, M., Roth, S. V., Gehrke, R., Stribeck, N., Almendarez-Camarillo, A. \& Lengeler, B. (2006). Appl. Phys. Lett. 88, 164102.

Stribeck, N. (2000). ACS Symp. Ser. 739, 41-56.

Stribeck, N. (2001). J. Appl. Cryst. 34, 496-503.

Stribeck, N. (2007). X-ray Scattering of Soft Matter. Berlin/Heidelberg/New York: Springer.

Stribeck, N., Nöchel, U. \& Almendarez-Camarillo, A. (2008b). Macromol. Chem. Phys. 209, 1976-1982.

Stribeck, N., Nöchel, U., Fakirov, S., Feldkamp, J., Schroer, C., Timman, A. \& Kuhlmann, M. (2008a). Macromolecules, 41, 76377647.

Vasanthan, N. (2004). Textile Res. J. 74, 545-550.

Vasanthan, N. (2007). J. Polym. Sci. B, 45, 349-357. 